\title{
Long noncoding RNA TCONS_00024652 regulates vascular endothelial cell proliferation and angiogenesis via microRNA-21
}

\author{
MUERTIZA HALIMULATI, BAGEDATI DUMAN, JULAITI NIJIATI and ABUDOUREYIMU AIZEZI \\ Department of Vascular and Thyroid Surgery, Center of Digestive and Vascular Surgery, \\ The First Affiliated Hospital of Xinjiang Medical University, Urumqi, Xinjiang 830054, P.R. China
}

Received October 19, 2017; Accepted January 19, 2018

DOI: $10.3892 /$ etm.2018.6594

\begin{abstract}
Acute coronary syndrome caused by the rupture of atherosclerotic plaques is one of the primary causes of major cardiovascular events, and neovascularization within the plaque is closely associated with its stability. Long noncoding RNA (lncRNAs) is a type of noncoding RNA that serves a crucial role in regulating vascular endothelial cells (VECs). The aim of the present study was to investigate the effect of lncRNA TCONS_00024652 on the proliferation and angiogenesis of VECs following stimulation with TNF- $\alpha$. The expression of lncRNA and miRNA was measured in human umbilical vein endothelial cells (HUVECs) by reverse transcription-quantitative polymerase chain reaction. Cell proliferation was measured using a Cell Counting Kit-8 assay. Wound healing and tube formation assays were performed to determine cell migration and angiogenesis. Interactions between TCONS_00024652 and miR-21 were determined using bioinformatics and a dual-luciferase reporter assay. The results demonstrated that TCONS_00024652 is highly expressed in TNF- $\alpha$-induced HUVECs. Functional assays demonstrated that the dysregulated expression of TCONS_00024652 promotes endothelial cell proliferation and angiogenesis, whereas TCONS_00024652 knockdown induces the opposite effects. Bioinformatics analysis using starBase predicted putative binding at the 3'-untranslated region of TCONS_00024652 and miR-21 and luciferase reporter assays further verified this interaction. The results of the present study suggest that the
\end{abstract}

Correspondence to: Professor Abudoureyimu Aizezi, Department of Vascular and Thyroid Surgery, Center of Digestive and Vascular Surgery, The First Affiliated Hospital of Xinjiang Medical University, 137 Carp Mountain Road, Urumqi, Xinjiang 830054, P.R. China

E-mail: abudoureyimu999@163.com

Abbreviations: lncRNA, long noncoding RNA; VECs, vascular endothelial cells; TNF- $\alpha$, tumor necrosis factor $\alpha$; HUVECs, human umbilical vein endothelial cells; PTEN, phosphatase and tensin homolog; TNFR1, tumor necrosis factor receptor 1

Key words: long non-coding RNA, atherosclerosis, vascular endothelial cells, angiogenesis, microRNA targeting of TCONS_00024652 by miR-21 may be a potential method of improving vascular endothelial dysfunction, neovascularization maturation and plaque stabilization.

\section{Introduction}

Angiogenesis is an important factor that influences the development of atherosclerotic plaques and is closely associated with local neovascularization and plaque stability (1). It has been demonstrated that excessive angiogenesis is associated with intraplaque hemorrhage, suggesting that neovascularization may contribute to lesion progression and rupture (2). Rupture-prone vulnerable plaques exhibit high lipid infiltration, increased numbers of inflammatory cells, a thin fibrous cap, and extensive endometrial and adventitial neovascularization (3-5). Studies have generally focused on the intimal accumulation of lipids and inflammatory cells $(6,7)$; however, the results of a recent study suggest that endothelial cells also serve a critical role during advanced plaque angiogenesis (8).

LncRNAs are transcripts that are $>200$ nucleotides long, which regulate transcription via chromatin modulation, post-transcriptional regulation, organization of protein complexes, cell signaling and allosteric regulation $(9,10)$. They serve important roles in physiological processes, including the differentiation, proliferation, apoptosis and invasion of induced pluripotent stem cells $(11,12)$. Studies have demonstrated that several lncRNAs, including lncRNA-p21 (13), metastasis associated lung adenocarcinoma transcript 1 (14) and lnc-Ang362 (15), are associated with the progression of atherosclerosis and relevant cellular processes. Furthermore, it has been confirmed that lncRNA is able to facilitate endothelial angiogenic function (16).

MicroRNAs (miRNAs) are endogenous small single-strand non-coding RNAs $\sim 22$ nucleotides long (17). Certain miRNAs are able to regulate angiogenic processes and negatively modulate angiogenesis (18). For example, miRs-221 and -222 are able to regulate the development and differentiation of endothelial cells (ECs) but suppress pro-angiogenic activation and migration (19). Furthermore, the EC-derived miRNA 17-92 cluster negatively regulates arteriogenesis via miRNA-19 repression of Wnt signaling (20). miR-21 is highly expressed in ECs and studies have demonstrated that miR-21 expression suppresses the expression of phosphatase and tensing homolog (PTEN) 
but upregulates vascular endothelial growth factor (VEGF) expression (21). Furthermore, miR-21 has been identified as a modulator of migration and tubulogenesis in HUVECs (22).

The present study measured the expression of lncRNAs in tumor necrosis factor (TNF)- $\alpha$-induced HUVECs and demonstrated that TCONS_00024652 was overexpressed. Functional experiments indicated that TCONS_00024652 silencing leads to the inhibition of angiogenesis and the expression of downstream miR-21. It was therefore suggested that TCONS_00024652 silencing induces the subsequent upregulation of miR-21.

\section{Materials and methods}

Cell culture. Human umbilical vein endothelial cells (HUVECs) were obtained from the American Type Culture Collection (cat. no. CRL-1730, Manassas, VA, America) and cultured in Dulbecco's modified Eagle's medium (DMEM; Gibco; Thermo Fisher Scientific, Inc., Waltham, MA, USA) with $10 \%(\mathrm{v} / \mathrm{v})$ fetal bovine serum (FBS; Hyclone; GE Healthcare, Logan, UT, USA) in a humidified incubator at $37^{\circ} \mathrm{C}$ with $5 \% \mathrm{CO}_{2}$. HUVECs were seeded at $3 \times 10^{5}$ cells/well in 6 -well plates and treated with varying concentrations $(0.5,1$ or $2 \mathrm{ng} / \mathrm{ml}$ for $24 \mathrm{~h}$ ) or for varying times ( $1 \mathrm{ng} / \mathrm{ml}$ for 6,12 or 24 h) with recombinant human TNF- $\alpha$ (Peprotech, Rock Hill, NJ, USA). Following treatment, cells were lysed and RNA or protein was extracted for further experiments.

Cell transfection. All small interfering (si)RNAs used in the current study were synthesized by Shanghai GenePharma Co., Ltd. (Shanghai, China), including si-lncRNA, mimic siRNAs, inhibitor siRNAs and scramble negative control (NC) siRNAs. Cells at $70 \%$ confluence were transfected with $100 \mathrm{nM}$ siRNAs using Lipofectamine 2000 (Invitrogen; Thermo Fisher Scientific, Inc.), following the manufacturer's protocol. The si-TCONS_00024652-1 sequences were: 5'-GUGAAU UCAACAGACUGAAUU-3' and the si-TCONS_00024652-2 sequence was 5'-GUGAUUUACCAGUCAGUGAAU-3'. The sequences of miR-21 mimics, inhibitor and negative control were as follow: miR-21 mimics forward, 5'-UGGCAGUCU CUUAGAUGGUGG-3' and reverse, 5'-GCCAUGCAAGAC ACUGCCAGG-3'; miR-21 inhibitor forward, 5'-UUCUAG CCUACACCGUCCCGAA-3' and reverse, 5'-UGGUAGUCG ACUCCGCACCTGA-3'; negative control forward: 5'-UUC UCGGAACGUGUCACGUTT-3' and reverse: 5'-UUCAAG GCCCGUGUCAUGUGG-3'. Subsequent experiments were performed $48 \mathrm{~h}$ following transfection.

Cell proliferation assay. EC proliferation was measured using a Cell Counting Kit-8 (CCK-8; Dojindo Molecular Technologies, Kuamoto, Japan). Following transfection, cells were seeded in 96-well plates at density of $5 \times 10^{3} /$ well. CCK- 8 solution was added and cells were incubated at $37^{\circ} \mathrm{C}$ for $90 \mathrm{~min}$. Subsequently, absorbance was measured using an Infinite 200 Pro microplate reader (Tecan Group Ltd., Mannedorf, Switzerland) at $450 \mathrm{~nm}$.

RNA extraction and reverse transcription-quantitative polymerase chain reaction $(R T-q P C R)$. Total RNA was extracted from HUVECs using total RNA extraction reagent (Shanghai Genepharma, Co. Ltd.) and cDNA was synthesized from the extracted RNA using a FastQuant RT kit [Tiangen Biotech (Beijing) Co., Ltd., Beijing, China]. qPCR was then performed using SuperReal PreMix Plus [Tiangen Biotech (Beijing), Co., Ltd., Beijing, China]. GAPDH was used as an internal control. All primers were synthesized by Sangon Biotech Co., Ltd. (Shanghai, China). The sequences of the primers used were as follows: miR-21 stem-loop RT primer, 5'-CTCAAC TGGTGTCGTGGAGTCGGCAATTCAGTTGAGTCAACA TC-3'; miR-21 forward, 5'-ACACTCCAGCTGGCTAGCTTA TCAGACTGATG-3' and reverse, 5'-CTCAACTGGTGTCGT GGA-3'; GAPDH forward, 5'-GTCAACGGATTTGGTCTG TATT-3' and 5'-AGTCTTCTGGGTGGCAGTGAT-3'. Primer sequences for lncRNA are listed in Table I. Thermal cycling conditions were as follows: $94^{\circ} \mathrm{C}$ for $15 \mathrm{~min}$; followed by 40 cycles of $94^{\circ} \mathrm{C}$ for $15 \mathrm{sec}, 60^{\circ} \mathrm{C}$ for $32 \mathrm{sec}, 72^{\circ} \mathrm{C}$ for $60 \mathrm{sec}$. Relative expression levels of gene were quantified based target gene/GAPDH and calculated using the $2^{-\Delta \Delta C q}$ method (23).

Bioinformatics analysis. RNA-RNA and protein-RNA interaction networks were analyzed using StarBase v2.0 (http://starbase.sysu.edu.cn/). The database contains unique features, including determination of ceRNA functional networks based on miRNA-target interactions, provides comprehensive miRNA-lncRNA interactions and drafts interaction maps between miRNAs and circRNAs (24).

Luciferase assay. To examine interactions between TCONS_00024652 and miR-21, wild-type pmirGLO-TCONS_00024652 and diverse mutant pmirGLO-TCONS_00024652 with pmirGLO-promotor vector (GenePharma, Shanghai, China) were constructed. miR-21 mimics, mimic NC, miR-21 inhibitor and inhibitor NC were purchased from GenePharma Co., Ltd. (Shanghai, China), 293T cells were plated at a density of $8 \times 10^{3}$ cells/well in 96 -well plates and cultured at $37^{\circ} \mathrm{C}$ with $5 \% \mathrm{CO}_{2}$ overnight. Cells were then co-transfected with the wild-type or mutant luciferase reporter plasmids, and miR-21 mimic or NC using Lipofectamine 2000. Following $48 \mathrm{~h}$, luciferase activity was measured using the Bright-Glo ${ }^{\mathrm{TM}}$ Luciferase assay system (Promega Corporation, Madison, WI, USA) and normalized to that of Renilla luciferase.

Wound healing assay. HUVECs at $80 \%$ confluence were plated at a density of $3 \times 10^{5}$ cells/well in 6 -well plates and cultivated in DMEM without FBS at $37^{\circ} \mathrm{C}$ overnight. The monolayer of HUVECs was scratched with a $1 \mathrm{ml}$ of a $200 \mu \mathrm{l}$ pipette tip to form wound gaps. Cells were washed by PBS and incubated with $1 \mathrm{ng} / \mathrm{ml} \mathrm{TNF}-\alpha$ at $37^{\circ} \mathrm{C}$ to induce cell migration for $24 \mathrm{~h}$. Images were captured using light microscope (magnification, $\mathrm{x} 200$ ). Cells were photographed prior to and $24 \mathrm{~h}$ following scratching and the migration distance were calculated by Image J 1.48 software (National Institutes of Health, Bethesda, MD, USA).

Tube formation assay. A tube formation assay was performed to detect the angiogenesis ability of HUVECs. Briefly, cells were harvested following treatment and seeded into a 96-well plate $\left(1 \times 10^{4}\right.$ cells/well) coated with $10 \mathrm{mg} / \mathrm{ml}$ Matrigel (BD Biosciences, Franklin Lakes, NJ, USA). Following $7 \mathrm{~h}$ incubation at $37^{\circ} \mathrm{C}$, capillary tube structures were observed and 
Table I. lcnRNA primers used in reverse transcription-quantitative polymerase chain reaction analysis.

Name

Sequence $\left(5^{\prime}-3^{\prime}\right)$

ATG9B forward

ATG9B reverse

LncRNA-p21 forward

LncRNA-p21 reverse

TCONS_00004897 forward

TCONS_00004897 reverse

TCONS_00024652 forward

TCONS_00024652 reverse

LNC00305 forward

LNC00305 reverse

SENCR forward

SENCR reverse

MALAT1 forward

MALAT1 reverse

ANRIL forward

ANRIL reverse

Tie-1AS forward

Tie-1AS reverse

TCONS_00004013 forward

TCONS_00004013 reverse

U6 forward

U6 reverse

GGTCTGCCATCTATTACTT
TCTACGCATTATAGTCACAA
ATAGTAGTTGGAGACTTCA
ACTGTATATTCAATGTTGGC
GGATGGCACTCCTAGTCTGC
GTGCAGGCCTGAGTCTTTA
ACGCTAACTGGCACCTTGTT
TGGGGATTACTGGGGTAGAT
TAAAGGATGCGTAGGGATGG
TTCATGATCACGCCCTCATA
TTTACTTTAACAGACCAGAA
CTCCTTTGTTGAATCCAT
TTATCCTTGGAAGAGTATT
TAAGAAGTCACATTATTGG
TTGATGAGAAGAATAAGCC
CTCCTTTGATGTGTGTTT
CCGGGGGTATACTACGGTC
CTCTAGAGGGGGTAGAGG
GCGCTAACTGGCACCTTGAT
TTTGGATTACTGGGGTATTG
GCTTCGGCAGCACATATACTAAAAT
CGCTTCACGAATTTGCGTGTCAT

MALAT1, metastasis-associated lung adenocarcinoma transcript 1; ANRIL, cyclin-dependent kinase inhibitor 2B antisense RNA 1; SENCR, smooth muscle and endothelial cell enriched migration/differentiation-associated lncRNA; tie-1AS, conserved natural noncoding antisense RNA in the tie-1 locus.

representative images were captured using a light microscope (magnification, $x 400)$. The branching length was determined using Image-Pro plus 6.0 (Media Cybernetics, Rockville, MD, USA).

Statistical analysis. All statistical analyses were performed using GraphPad Prism 6.0 software (GraphPad, Software Inc., La Jolla, CA, USA) and SPSS version 19.0 (IBM Corp., Armonk, NY, USA). Unpaired Student's t test or one-way analysis of variance followed by least significant difference tests were performed to determine differences among groups. Heterogeneous data was analyzed using one-way analysis of variance followed by Dunnett's tests. All data are presented as the mean \pm standard deviation and $\mathrm{P}<0.05$ was considered to indicate statistically significant difference.

\section{Results}

LncRNA expression profiles indicate that TCONS_00024652 is upregulated following TNF-a treatment in HUVECs. During the initial phase of the current study, HUVECs were stimulated with TNF- $\alpha$ and levels of lncRNAs associated with the regulation of vascular function $(25,26)$ and atherosclerosis (27) were assessed using RT-qPCR. The results revealed that expression of a majority of lncRNAs was significantly increased in these HUVECs following TNF- $\alpha$ treatment;
TCONS_00024652 exhibited the largest changes (Fig. 1A). Therefore, it was selected as the target lncRNA in subsequent experiments. RT-qPCR was performed to further validate the up-regulation of TCONS_00024652 following stimulation with TNF- $\alpha$ (Fig. 1B and C). These results indicate that TNF- $\alpha$ treatment up-regulates the expression of TCONS_00024652 in a dose-dependent manner.

TCONS_00024652 knockdown inhibits the proliferation, migration and angiogenesis of HUVECs. The function of TCONS_00024652 in cell proliferation and angiogenesis was investigated. To ensure the effectiveness of siRNAs in gene inhibition, two TCONS_00024652 siRNAs (si-TCONS_00024652-1 and si-TCONS_00024652-2) were designed and transfected into HUVECs to determine the effect of TCONS_00024652 on atherosclerosis progression. Transfection efficiency was determined using RT-qPCR and the results indicated that the transfection efficiency of si-TCONS_00024652-1 was higher than that of si-TCONS_0024652-2 (Fig. 2A). Furthermore, the results of the CCK-8 assay indicated that si-TCONS_00024652-1 was more effective at reducing the proliferation of HUVECs compared with si-TCONS_00024652-2 (Fig. 2B). Therefore, si-TCONS_00024652-1 alone was used in subsequent experiments. The results of the wound healing assay indicated that TCONS_00024652 silencing significantly decreased 
A

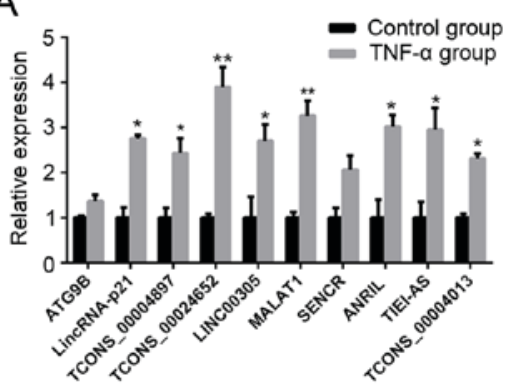

B

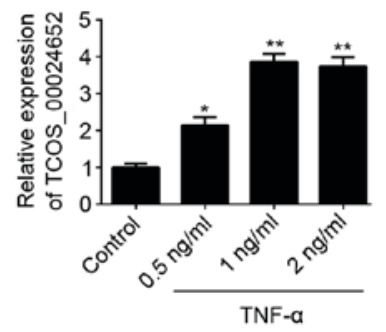

C

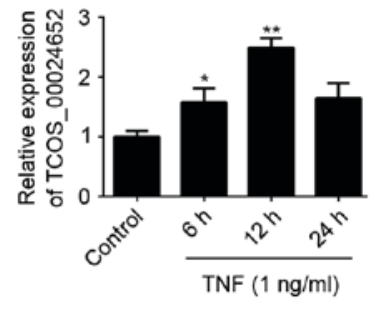

Figure 1. The expression of TCONS_00024652 was significantly increased in TNF- $\alpha$-induced HUVECs. (A) Following stimulation with TNF- $\alpha$ for $24 \mathrm{~h}$, the relative expression profile of IncRNA in HUVECs was detected by reverse transcription-quantitative polymerase chain reaction. (B) Relative expression of TCONS_00024652 in HUVECs following treatment with different concentrations $(0,0.5,1$ and $2 \mathrm{ng} / \mathrm{ml})$ of TNF- $\alpha$. (C) Relative expression of TCONS_00024652 in HUVECs following treatment with $1 \mathrm{ng} / \mathrm{ml} \mathrm{TNF}-\alpha$ over different time periods $(0,6,12$ and $24 \mathrm{~h})$. Data are presented as the mean \pm standard deviation. ${ }^{*} \mathrm{P}<0.05$ and ${ }^{* *} \mathrm{P}<0.01$ vs. control. HUVEC, human umbilical vein endothelial cells; TNF, tumor necrosis factor; lncRNA, long non-coding RNA.
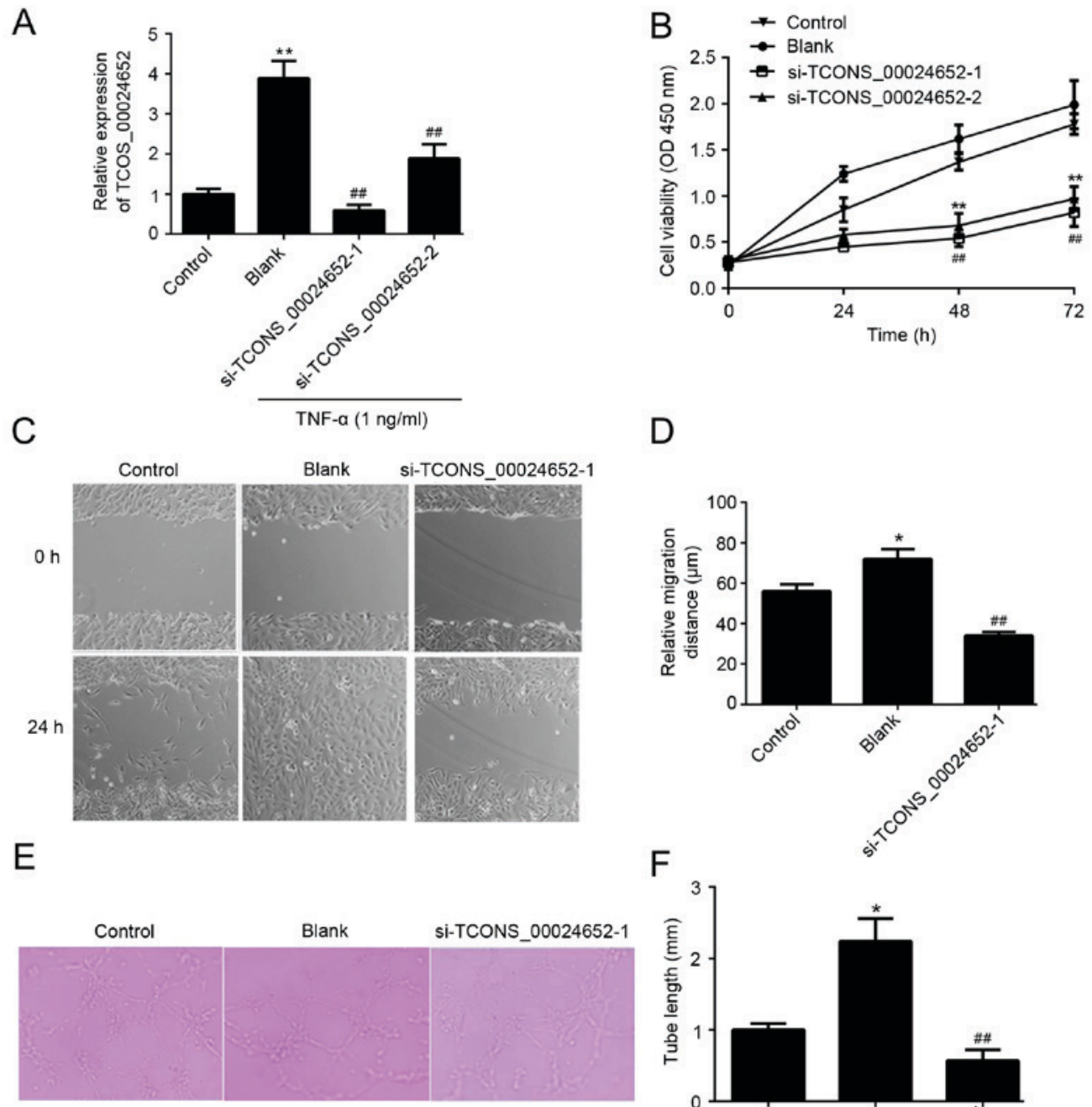

D
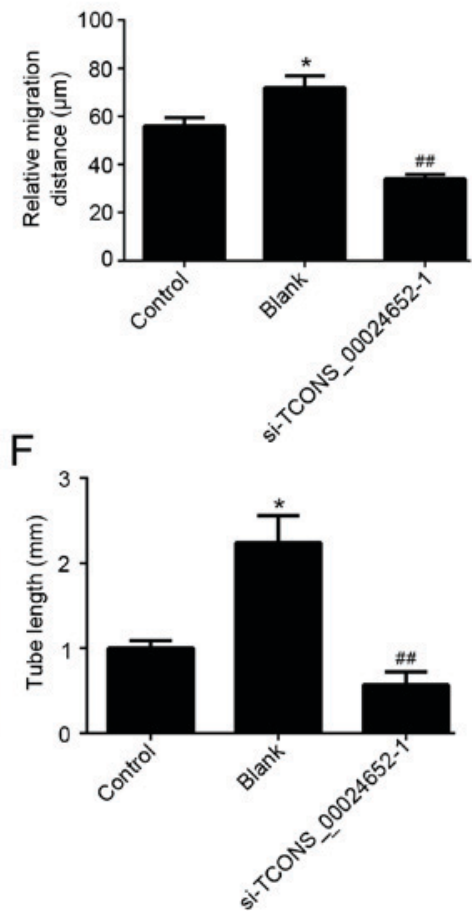

Figure 2. TCONS_00024652 promotes the proliferation, migration and angiogenesis of HUVECs in vitro. (A) The relative expression of TCONS_00024652 in HUVECs following transfection with si-NC, si-TCONS_00024652-1 or si-TCONS_00024652-2 was measured using reverse transcription-quantitative polymerase chain reaction. (B) A Cell Counting Kit-8 assay was used $24 \mathrm{~h}$ following transfection with si-TCONS_00024652-1 and si-TCONS_00024652-2 to determine the proliferation of HUVECs. (C) The migratory ability of HUVECs was detected using a wound-healing assay following transfection with si-TCONS_00024652-1 (magnification, x200). (D) Histogram presenting relative migration distance. (E) Transfected HUVECs were seeded onto Matrigel and then allowed to form capillary-like structures for $7 \mathrm{~h}$ (magnification, $\mathrm{x} 400$ ). (F) Statistical analysis of the length of tubular structures in each group. Data are presented as the mean \pm standard deviation. ${ }^{*} \mathrm{P}<0.05$ and ${ }^{* *} \mathrm{P}<0.01$ vs. control; ${ }^{\# *} \mathrm{P}<0.01$ vs. blank. Si, small interfering; HUVEC, human umbilical vein endothelial cells; TNF, tumor necrosis factor; OD, optical density. 
A

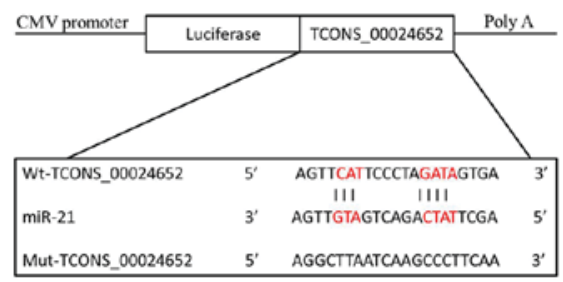

C

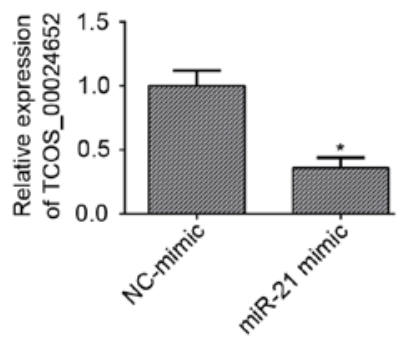

B

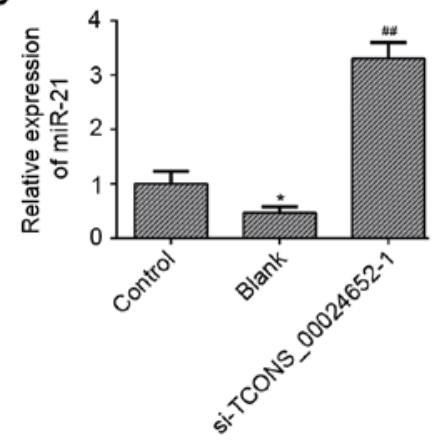

E

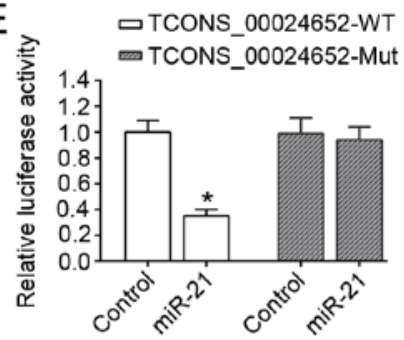

Figure 3. miR-21 is a direct target of TCONS_00024652 in HUVECs. (A) Putative complementary sites within miR-21 and the 3'-untranslated region of TCONS_00024652 were predicted using starBase. (B) Relative expression of miR-21 in HUVECs transfected with si-TCONS_00024652. (C and D) Relative expression of TCONS_00024652 mRNA levels following transfection with miR-21 mimic or inhibitor, as determined using reverse transcription-quantitative polymerase chain reaction. (E) Luciferase activity in HUVECs following co-transfection with miR-21 and luciferase reporters containing WT- or mut-TCONS_00024652 transcript. Data are presented as the mean \pm standard deviation. ${ }^{*} \mathrm{P}<0.05$ vs. control. ${ }^{\# \#} \mathrm{P}<0.01$ vs. blank. miR-21, microRNA-21; si, small interfering; NC, negative control; Mut, mutant; WT, wild-type; HUVEC, human umbilical vein endothelial cells.

the migration of cells compared with the blank control (Fig. 2C and D). Furthermore, TCONS_00024652 knockdown significantly reduced the ability of endothelial cells to form lumens (Fig. 2E and F). Taken together, these results indicate that TCONS_00024652 promotes the proliferation and angiogenesis of endothelial cells.

TCONS_00024652 directly targets miR-21. Previous studies have demonstrated that miR-21 possesses anti-proliferative $(28)$ and anti-angiogenesis $(24,29)$ properties; therefore it was hypothesized that miR-21 may be a downstream target in TNF- $\alpha$-induced HUVEC activation. Bioinformatics analysis indicated that miR-21 was a potential target of TCONS_00024652 (Fig. 3A). To explore the effect of TCONS_00024652 on miR-21, its expression was measured in HUVECs following TCONS_00024652 knockdown. The results demonstrated that miR-21 expression was significantly increased compared with the blank control (Fig. 3B). Furthermore, the expression of TCONS_00024652 was significantly decreased in HUVECs transfected with the miR-21 mimic (Fig. 3C) and increased in cells transfected with the miR-21 inhibitor (Fig. 3D). The results of the luciferase reporter assay confirmed that miR-21 interacts with TCONS_00024652, indicating that luciferase activity was significantly decreased following transfection of miR-21 into TCONS_0024652-WT (Fig. 3E). Overall, these data indicate that TCONS_00024652 downregulates miR-21 expression.

miR-21 inhibition reverses the inhibitory effect of TCONS_00024652 knockdown on proliferation, migration and angiogenesis in HUVECs. The aforementioned results demonstrated that there is an association between TCONS_00024652 and miR-21. Thus, it was hypothesized that miR-21 may neutralize the effects of TCONS_00024652 on HUVECs. A series of experiments were performed to confirm that TCONS_00024652 affects cell proliferation and angiogenesis by regulating miR-21 expression in endothelial cells. In cells stably transfected with si-TCONS_00024652, the expression of miR-21 was significantly higher than in the control group; this increase was reversed following transfection with miR-21 inhibitor (Fig. 4A). The co-transfection of the miR-21 inhibitor significantly reversed the inhibitory effects of si-TCONS_00024652 on HUVEC proliferation, migration and invasion (Fig. 4B-D). These results demonstrate that TCONS_00024652 affects endothelial cell proliferation and angiogenesis via the regulation of miR-21 expression, suggesting that TCONS_00024652 acts as a competing endogenous RNA (ceRNA) for miR-21.

\section{Discussion}

Studies investigating the effects of inflammation on atherosclerotic plaques have tended to focus on their innate immune and adaptive immune responses. However, it has been demonstrated that inflammation is closely associated with neovascularization in plaques and that this may be another mechanism which increases the vulnerability of plaques (30). Chemokine and inflammatory factors secreted by plaque inflammatory cells are able to directly or indirectly promote angiogenesis; this process is referred to as 'inflammatory angiogenesis' (31). The biological effect of TNF- $\alpha$ depends on its concentration and the type of cell it is acting on. High concentrations of TNF- $\alpha$ 
A

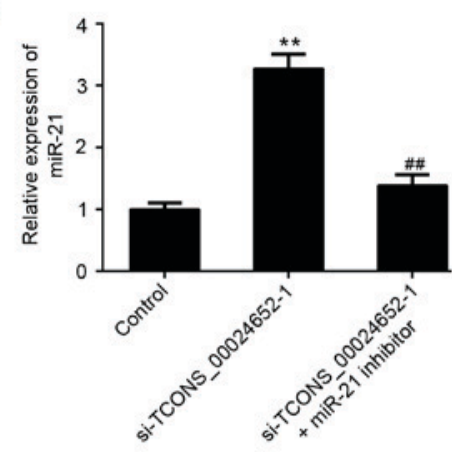

B

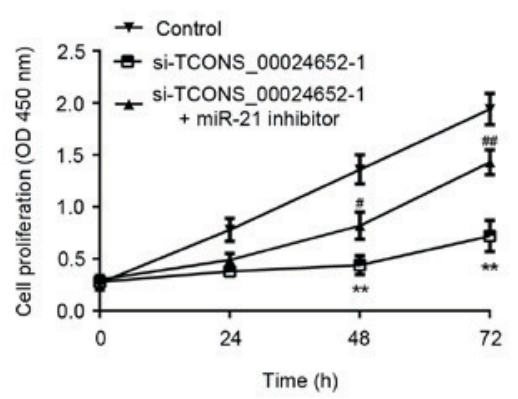

C
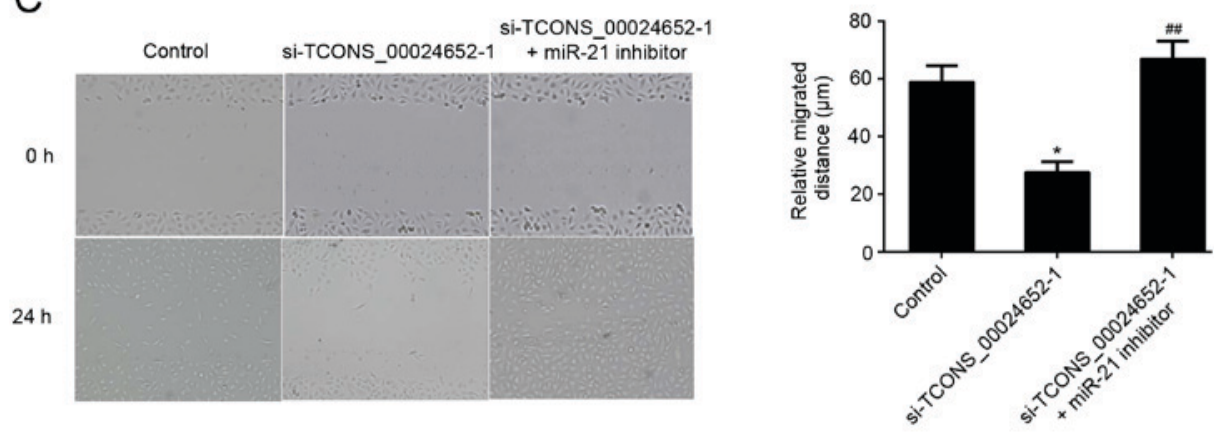

D

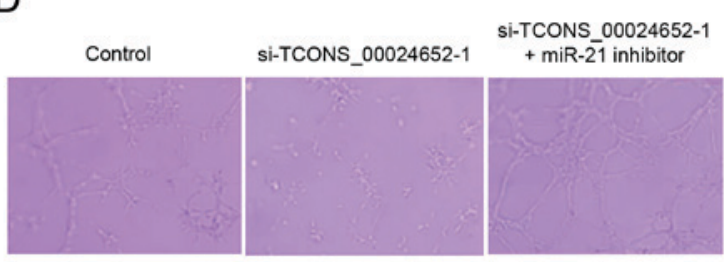

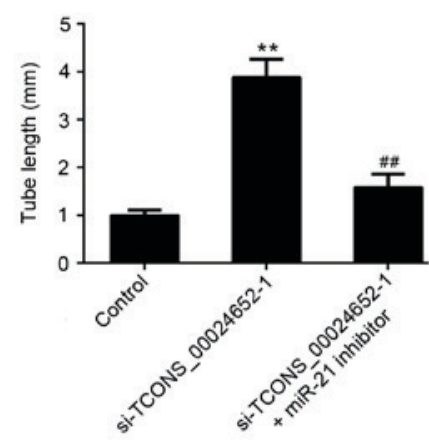

Figure 4.miR-21 inhibitor reversed the suppression of HUVEC proliferation, migration and angiogenesis following TCONS_00024652 knockdown. (A) Relative expression of miR-21 in HUVECs following transfection with si-TCONS_00024652 and miR-21 inhibitor, as determined by reverse transcription-quantitative polymerase chain reaction (B) miR-21 inhibitor reversed the si-TCONS_00024652-induced inhibition of proliferation, as determined by a Cell Counting Kit-8 assay. (C) Wound healing assay was performed following transfection with si-TCONS_00024652 and miR-21 inhibitor and relative migration distance was calculated (magnification, x200) (D) Transfected HUVECs were seeded onto Matrigel and incubated for $7 \mathrm{~h}$; representative image and tube length analysis are presented (magnification, $\mathrm{x} 400$ ). Data are presented as the mean \pm standard deviation. ${ }^{*} \mathrm{P}<0.05$ and ${ }^{* *} \mathrm{P}<0.01$ vs. control. ${ }^{\#} \mathrm{P}<0.05$ and ${ }^{\# \#} \mathrm{P}<0.01$ vs. si-TCONS_00024652-1. Si, small interfering; miR-21, microRNA-21; HUVEC, human umbilical vein endothelial cells; OD, optical density.

activate tumor necrosis factor receptor (TNFR) 1 to induce inflammation and stimulate the apoptosis of endothelial cells; however low concentrations of TNF- $\alpha$ promote endothelial cell proliferation, migration and microvascular production via TNFR2 (32). The present study successfully used low concentrations of TNF- $\alpha$ to induce endothelial angiogenesis and also confirmed the important role that inflammation serves in angiogenesis.

Previous studies have demonstrated the critical role that lncRNAs serve during the progression of various diseases $(33,34)$, including cardiovascular disease (35). It has been demonstrated that smooth muscle and endothelial cell enriched migration/differentiation-associated lncRNA regulates endothelial cell differentiation and the angiogenic capacity of HUVEC (36). Furthermore, a conserved natural noncoding antisense RNA in the tie-1 locus
lncRNA-myocardial infarction associated transcript serve important roles in the pathological mechanism of atherosclerosis and may function as molecular sponges in VECs $(37,38)$. In the present study, the gene expression profile of the lncRNA TCONS_00024652 was screened and its functional importance in TNF- $\alpha$-induced HUVECs was determined. TCONS_00024652 silencing reduced the proliferation and angiogenesis of TNF- $\alpha$-induced HUVECs, suggesting that TCONS_00024652 serves an angiogenesis-promoting role in TNF- $\alpha$-stimulated HUVECs.

In addition to regulating transcription, it has been demonstrated that many lncRNAs function as endogenous decoys for miRNAs. For example, LOC100129973, an important regulator of endothelial cell apoptosis, contains sites that bind to miR-4707-5p and miR-4767 (39). XLOC_008466 functions as an oncogene in non-small cell lung cancer by regulating 
the miR-874-matrix metalloproteinase/X-linked inhibitor of apoptosis protein axis (40). In the present study, bioinformatics was used to search for target miRNAs to reveal the downstream regulatory pathway by which TCONS_00024652 acts on HUVECs. The results indicated that miR-21 directly targets TCONS_00024652. The rescue experiments and luciferase reporter assay confirmed that TCONS_00024652 and miR-21 had a pairing region. Furthermore, it was demonstrated that TCONS_00024652 targets miR-21, decreasing its expression, thus acting as a ceRNA or molecular sponge. This indicates that TCONS_00024652 induces the proliferation and angiogenesis of VECs and stimulates the formation of atherosclerotic plaques. Although the results of the present study clearly indicate that there is an interaction between miR-21 and TCONS_00024652 that results in the regulation of HUVEC proliferation and angiogenesis, their effects on downstream target proteins remains uncertain. The instability of atherosclerotic plaques is associated with immature angiogenesis and the regulation of the interaction between VEGF and other factors may result in the recruitment of pericytes and promotion of neovascularization by the basement membrane (41). Future studies are required to elucidate the mechanisms of action of TCONS_00024652 and miR-21 on atherosclerotic plaques.

In conclusion, the results of the current study indicate that the increased expression of the endothelium-associated IncRNA TCONS_00024652 promotes plaque angiogenesis and the progression of atherosclerosis via the TCONS_00024652/miR-21 signaling pathway, suggesting that TCONS_00024652 may be an important molecular marker that stabilizes atherosclerotic plaques and slows down the progression of atherosclerosis.

\section{Acknowledgements}

The authors wish to thank Dr. Liu from the Basic Medical Research Center of Xinjiang Medical University for his technical support throughout the study.

\section{Funding}

No funding was received.

\section{Availability of data and materials}

The datasets used and/or analyzed during the current study are available from the corresponding author on reasonable request.

\section{Authors' contributions}

MH performed the experiments and prepared the manuscript. AA designed and organized the project and helped with the preparation of the manuscript. BD analyzed the data and performed statistics. JN collected and interpreted the data. All authors read and approved the final manuscript.

\section{Ethics approval and consent to participate}

Not applicable.

\section{Patient consent for publication}

Not applicable.

\section{Competing interests}

The authors declare that they have no competing interests.

\section{References}

1. Camaré C, Pucelle M, Nègre-Salvayre A and Salvayre R: Angiogenesis in the atherosclerotic plaque. Redox Biol 12: 18-34, 2017.

2. Weng J: Activation of CD137 signaling promotes angiogenesis in atherosclerosis via modulating endothelial Smad1/5-NFATc1 pathway. J Am Heart Assoc 6: 234-247, 2017.

3. Sun Z: Atherosclerosis and Atheroma Plaque Rupture: Imaging Modalities in the Visualization of Vasa Vasorum and Atherosclerotic Plaques. Sci World J 23: 1-12, 2014.

4. Mulligan-Kehoe MJ: The vasa vasorum in diseased and nondiseased arteries. Am J Physiol Heart Circ Physiol 298: H295-H305, 2010.

5. Moguillansky D, Leng X, Carson A, Lavery L, Schwartz A, Chen X and Villanueva FS: Quantification of plaque neovascularization using contrast ultrasound: A histologic validation. Eur Heart J 32: 646-653, 2011.

6. Wu MY and Li CJ: New insights into the role of inflammation in the pathogenesis of atherosclerosis. Int J Mol Sci 18: E2034, 2017.

7. Pirillo A, Bonacina F, Norata GD and Catapano AL: The interplay of lipids, lipoproteins and immunity in atherosclerosis. Curr Atheroscler Rep 20: 12, 2018.

8. Sedding DG, Boyle EC, Demandt JAF, Sluimer JC, Dutzmann J, Haverich A and Bauersachs J: Vasa vasorum angiogenesis: Key player in the initiation and progression of atherosclerosis and potential target for the treatment of cardiovascular disease. Front Immunol 9: 706, 2018

9. Tsai MC, Manor O, Wan Y, Mosammaparast N, Wang JK, Lan F, Shi Y, Segal E and Chang HY: Long Noncoding RNA as modular scaffold of histone modification complexes. Science 329: 689-693, 2010.

10. Schmitz SU, Grote P and Herrmann BG: Mechanisms of long noncoding RNA function in development and disease. Cell Mol Life Sci 73: 2491-2509, 2016.

11. Taylor DH, Chu ETJ, Spektor R and Soloway PD: Long non-coding RNA regulation of reproduction and development. Mol Reprod Dev 82: 932-956, 2015.

12. Hecht PM, Ballesteros-Yanez I, Grepo N, Knowles JA and Campbell DB: Noncoding RNA in the transcriptional landscape of human neural progenitor cell differentiation. Front Neurosci 9: 392, 2015.

13. Wu G, Cai J, Han Y, Chen J, Huang ZP, Chen C, Cai Y, Huang H, Yang Y, Liu Y, et al: LincRNA-p21 regulates neointima formation, vascular smooth muscle cell proliferation, apoptosis and atherosclerosis by enhancing p53 activity. Circulation 130: 1452-1465, 2014.

14. Puthanveetil P, Chen S, Feng B, Gautam A and Chakrabarti S: Long non-coding RNA MALAT1 regulates hyperglycaemia induced inflammatory process in the endothelial cells. J Cell Mol Med 19: 1418-1425, 2015.

15. Leung A, Trac C, Jin W, Lanting L, Akbany A, Sætrom P, Schones DE and Natarajan R: Novel long non-coding RNAs are regulated by angiotensin II in vascular smooth muscle cells. Circ Res 113: 266-278, 2013.

16. Leisegang MS, Fork C, Josipovic I, Richter FM, Preussner J, Hu J, Miller MJ, Epah J, Hofmann P, Günther S, et al: Long noncoding RNA MANTIS facilitates endothelial angiogenic function. Circulation 136: 65-79, 2017.

17. Bhaskaran M and Mohan M: MicroRNAs: History, biogenesis and their evolving role in animal development and disease. Vet Pathol 51: 759-774, 2014.

18. Chen LJ, Lim SH, Yeh YT, Lien SC and Chiu JJ: Roles of microRNAs in atherosclerosis and restenosis. J Biomed Sci 19: 79-91, 2012.

19. Chistiakov DA, Sobenin IA, Orekhov AN and Bobryshev YV: Human miR-221/222 in physiological and atherosclerotic vascular remodeling. Biomed Res Int 15: 1216-1229, 2015. 
20. Landskroner-Eiger S, Qiu C, Perrotta P, Siragusa M, Lee MY, Ulrich V, Luciano AK, Zhuang ZW, Corti F, Simons M, et al: Endothelial miR-17 92 cluster negatively regulates arteriogenesis via miRNA-19 repression of WNT signaling. Proc Natl Acad Sci USA 112: 12812-12817, 2015.

21. Haque R, Iuvone PM, He L, Choi KSC, Ngo A, Gokhale S, Aseem $M$ and Park D: The MicroRNA-21 signaling pathway is involved in prorenin receptor (PRR)-induced VEGF expression in ARPE-19 cells under a hyperglycemic condition. Mol Vision 23: 251-262, 2017.

22. Luo M, Tan X, Mu L, Luo Y, Li R, Deng X, Chen N, Ren M, Li Y, Wang L, et al: MiRNA-21 mediates the antiangiogenic activity of metformin through targeting PTEN and SMAD7 expression and PI3K/AKT pathway. Sci Rep 7: 4521-4534, 2017.

23. Livak KJ and Schmittgen TD: Analysis of relative gene expression data using real-time quantitative PCR and the 2(-Delta Delta C(T)) method. Methods 25: 402-408, 2001.

24. Li JH, Liu S, Zhou H, Qu LH and Yang JH: starBase v2.0 Decoding miRNA-ceRNA, miRNA-ncRNA and protein-RNA interaction networks from large-scale CLIP-Seq data. Nucleic Acids Res 42: D92-D97, 2014.

25. Uchida S and Dimmeler S: Long noncoding RNAs in cardiovascular diseases. Circ Res 116: 737-750, 2015.

26. Zhang BY, Jin Z and Zhao Z: Long intergenic noncoding RNA 00305 sponges miR-136 to regulate the hypoxia induced apoptosis of vascular endothelial cells. Biomed Pharmacother 94: 238-243, 2017

27. Li H, Zhu H and Ge J: Long noncoding RNA: Recent updates in atherosclerosis. Int J Biol Sci 12: 898-910, 2016.

28. Xu L, Wu Z, Chen Y, Zhu Q, Hamidi S and Navab R: MicroRNA-21 (miR-21) regulates cellular proliferation, invasion, migration and apoptosis by targeting PTEN, RECK and Bcl-2 in lung squamous carcinoma, Gejiu City, China. PLoS One 9 5324-5337, 2014

29. Sabatel C, Malvaux L, Bovy N, Deroanne C, Lambert V, Gonzalez ML, Colige A, Rakic JM, Noël A, Martial JA and Struman I: MicroRNA-21 exhibits antiangiogenic function by targeting rhob expression in endothelial cells. PLoS One 6: 6548-6560, 2011.

30. Osborn EA and Jaffer FA: Imaging inflammation and neovascularization in atherosclerosis: Clinical and translational molecular and structural imaging targets. Curr Opin Cardiol 30: 671-680, 2015.

31. De León H, Boué S, Schlage WK, Boukharov N, Westra JW, Gebel S, VanHooser A, Talikka M, Fields RB, Veljkovic E, et al: A vascular biology network model focused on inflammatory processes to investigate atherogenesis and plaque instability. J Transl Med 12: 185, 2014
32. Luo D, Luo Y, He Y, Zhang H, Zhang R, Li X, Dobrucki WL, Sinusas AJ, Sessa WC and Min W: Differential functions of tumor necrosis factor receptor 1 and 2 signaling in ischemia-mediated arteriogenesis and angiogenesis. Am J Pathol 169: 1886-1898, 2006.

33. Ulitsky I and Bartel DP: lincRNAs: Genomics, evolution and mechanisms. Cell 154: 26-46, 2013.

34. Di Gesualdo F, Capaccioli S and Lulli M: A pathophysiological view of the long non-coding RNA world. Oncotarget 5: 10976-10996, 2014.

35. Gomes CPC, Spencer H, Ford KL, Michel LYM, Baker AH, Emanueli C, Balligand JL and Devaux Y; Cardiolinc network: The function and therapeutic potential of long Non-coding RNAs in cardiovascular development and disease. Mol Ther Nucleic Acids 8: 494-507, 2017.

36. Boulberdaa M, Scott E, Ballantyne M, Garcia R, Descamps B, Angelini GD, Brittan M, Hunter A, McBride M, McClure J, et al: A role for the Long Noncoding RNA SENCR in commitment and function of endothelial cells. Mol Ther 24: 978-990, 2016.

37. Yan B, Yao J, Liu JY, Li XM, Wang XQ, Li YJ, Tao ZF, Song YC, Chen Q and Jiang Q: IncRNA-MIAT regulates microvascular dysfunction by functioning as a competing endogenous RNA. Circ Res 116: 1143-1156, 2015.

38. Li K, Blum Y, Verma A, Liu Z, Pramanik K, Leigh NR, Chun CZ, Samant GV, Zhao B and Garnaas MK, et al: A noncoding antisense RNA in tie-1 locus regulates tie-1 function in vivo. Blood 115: 133-139, 2010.

39. Lu W, Huang SY, Su L, Zhao BX and Miao JY: Long Noncoding RNA LOC100129973 suppresses apoptosis by targeting miR-4707-5p and miR-4767 in vascular endothelial cells. Sci Rep 6: 9533-9547, 2016.

40. Yang R, Li P, Zhang G, Lu C, Wang $\mathrm{H}$ and Zhao G: Long non-coding RNA XLOC_008466 functions as an oncogene in human non-small cell lung cancer by targeting miR-874. Cell Physiol Biochem 42: 126-136, 2017.

41. Cerezo AB, Hornedo-Ortega R, Alvarez-Fernandez MA, Troncoso AM and Garcia-Parrilla MC: Inhibition of VEGF-induced VEGFR-2 activation and HUVEC migration by melatonin and other bioactive indolic compounds. Nutrients 9: E249, 2017. 